\title{
Almost Condensed Domains
}

\author{
Waseem Khalid and Shafiq Ur Rehman
}

\begin{abstract}
As an extension of the class of half condensed domains introduced by D.D. Anderson and Dumitrescu, we introduce and study the class of almost condensed domains. An integral domain $D$ is almost condensed if whenever $0 \neq z \in I J$ with $I, J$ ideals of $D$, there exist $I^{\prime}, J^{\prime}$ ideals of $D$ such that $I^{\prime} \subseteq I_{w}, J^{\prime} \subseteq J_{w}$ and $z D=\left(I^{\prime} J^{\prime}\right)_{w}$.
\end{abstract}

In 1983, D.F. Anderson and D.E. Dobbs [13] called an integral domain $D$ condensed if for each pair of ideals $I, J$ of $D, I J=\{i j \mid i \in I, j \in J\}$. A Bézout domain is condensed, cf. [13, Corollary 2.2] and an integrally closed condensed domain is Bézout, cf. [11, Main Theorem]. In 2004, D.D. Anderson and T. Dumitrescu [6] called an integral domain $D$ a half condensed (HC) domain if whenever $0 \neq z \in I J$ with $I, J$ ideals of $D$, there exist $I^{\prime}, J^{\prime}$ (invertible) ideals of $D$ such that $I^{\prime} \subseteq I, J^{\prime} \subseteq J$ and $z D=I^{\prime} J^{\prime}$. In this paper we study the following extension of the concept of $\mathrm{HC}$ domain. We call an integral domain $D$ an almost condensed $(A C)$ domain if whenever $0 \neq z \in I J$ with $I, J$ ideals of $D$, there exist $I^{\prime}, J^{\prime}$ (w-invertible) ideals of $D$ such that $I^{\prime} \subseteq I_{w}, J^{\prime} \subseteq J_{w}$ and $z D=\left(I^{\prime} J^{\prime}\right)_{w}$ (the definition of the $w$-closure of an ideal as well as other basic facts are recalled below). The following implications hold.

Key Words: Condensed domain, Half condensed domain, Prüfer v-multiplication domain. 2010 Mathematics Subject Classification: Primary 46G05, 46L05; Secondary 47A30, $47 \mathrm{~B} 47$

Received: October, 2011.

Revised: November, 2011.

Accepted: November, 2012. 


\begin{tabular}{|c|c|c|c|c|}
\hline Bézout domain & $\Rightarrow_{1}$ & Prüfer domain & $\Rightarrow_{2}$ & PVMD \\
\hline$\Downarrow_{3}$ & & $\Downarrow_{4}$ & & $\Downarrow_{5}$ \\
\hline
\end{tabular}

The implications " 1 " and " 2 " are obvious, " 3 " is proved in [13, Corollary 2.2], " 4 " is proved in [6, Proposition 1.2], " 5 " is proved in Theorem 4 of this paper and "6", "7" are clear from definitions.

This new concept of AC domain depends upon the notion of star operation, $v$-operation, $t$-operation and $w$-operation. A reader in need of a quick review on this topic may consult sections 32 and 34 of Gilmer's book [18]. For the reader's convenience we give a working introduction here for the notions involved. Let $D$ be an integral domain with quotient field $K$ and let $F(D)$ denote the set of nonzero fractional ideals of $D$. A function $A \mapsto A^{*}: F(D) \rightarrow F(D)$ is called a star operation on $D$ if $*$ satisfies the following three conditions for all $0 \neq a \in K$ and for all $I, J \in F(D):(1) D^{*}=D$ and $(a I)^{*}=a I^{*},(2) I \subseteq I^{*}$ and if $I \subseteq J$, then $I^{*} \subseteq J^{*},(3)\left(I^{*}\right)^{*}=I^{*}$. An ideal $I \in F(D)$ is called a $*-$ ideal if $I^{*}=I$. For all $I, J \in F(D)$, we have $(I J)^{*}=\left(I^{*} J\right)^{*}=\left(I^{*} J^{*}\right)^{*}$. These equations define the so-called $*$-multiplication. If $\left\{I_{\alpha}\right\}$ is a subset of $F(D)$ such that $\cap I_{\alpha} \neq 0$, then $\cap I_{\alpha}^{*}$ is a $*$-ideal. Also, if $\left\{I_{\alpha}\right\}$ is a subset of $F(D)$ such that $\sum I_{\alpha}$ is a fractional ideal, then $\left(\sum I_{\alpha}\right)^{*}=\left(\sum I_{\alpha}^{*}\right)^{*}$. A star operation * is said to be a stable star operation if $(I \cap J)^{*}=I^{*} \cap J^{*}$ for all $I, J \in F(D)$. The function $*_{f}: F(D) \rightarrow F(D)$ given by $I^{*_{f}}=\cup J^{*}$, where $J$ ranges over all nonzero finitely generated sub-ideals of $I$, is also a star operation; $*$ is said to be a star operation of finite character if $*=*_{f}$. Clearly $\left(*_{f}\right)_{f}=*_{f}$. Let $\operatorname{Max}_{*}(D)$ denote the set of maximal $*$-ideals, that is, ideals maximal among proper integral $*$-ideals of $D$. Every maximal $*$-ideal is a prime ideal. If $*$ is of finite character, then every proper $*$-ideal is contained in some maximal $*$-ideal, and $*$ is stable if and only if $I^{*}=\cap_{P \in \operatorname{Max}_{*}(D)} I D_{P}$ for all $I \in F(D)$, cf. [1, Corollary 4.2]. A *-ideal $I$ is of finite type if $I=\left(a_{1}, \ldots, a_{n}\right)^{*}$ for some $a_{1}, \ldots, a_{n} \in I$. An ideal $I \in F(D)$ is said to be $*$-invertible if $\left(I I^{-1}\right)^{*}=D$, where $I^{-1}=(D: I)=\{x \in K \mid x I \subseteq D\}$. If $*$ is of finite character, then $I$ is *-invertible if and only if $I I^{-1}$ is not contained in any maximal *-ideal of $D$; in this case $I^{*}=\left(a_{1}, \ldots, a_{n}\right)^{*}$ for some $a_{1}, \ldots, a_{n} \in I$.

Let $*_{1}, *_{2}$ be star operations on $D$. We write $*_{1} \leq *_{2}$, if $I^{*_{1}} \subseteq I^{*_{2}}$ for all $I \in F(D)$. In this case we get $\left(I^{*_{1}}\right)^{*_{2}}=I^{*_{2}}=\left(I^{*_{2}}\right)^{*_{1}}$ and every $*_{1}$-invertible ideal is $*_{2}$-invertible. Some well-known star operations are: the $d$-operation 
(given by $I \mapsto I$ ), the $v$-operation (given by $I \mapsto I_{v}=\left(I^{-1}\right)^{-1}$ ) and the $t$ operation (defined by $t=v_{f}$ ). The $w$-operation is the star operation given by $I \mapsto I_{w}=\{x \in K \mid J x \subseteq I$ for some finitely generated ideal $J$ of $D$ with $\left.J^{-1}=D\right\}$. The $w$-operation is a stable star operation of finite character. For every $I \in F(D)$, we have $I \subseteq I_{w} \subseteq I_{t} \subseteq I_{v}$; so a $v$-ideal is a $t$-ideal and a $t$-ideal is a $w$-ideal. It is known that $\operatorname{Max}_{w}(D)=\operatorname{Max}_{t}(D)$, cf. [3, Corollary 2.17] and $I_{w}=\cap_{P \in \operatorname{Max}_{t}(D)} I D_{P}$ by [3, Corollary 2.13]. So, a nonzero fractional ideal is $w$-invertible if and only if it is $t$-invertible. Recall from [15] that the quotient group of $t$-invertible $t$ - fractional ideals modulo the subgroup of principal fractional ideals is called the $t$-class group denoted by $C l_{t}(D)$.

Let $*$ be a finite character star operation on an integral domain $D$. Recall from [2] that $D$ is called $*$-Noetherian if $D$ satisfies the ascending chain condition for $*$-ideals (equivalently, if for every ideal $I$ there exists a finitely generated ideal $J \subseteq I$ with $I^{*}=J^{*}$ ). For instance, the $t$-Noetherian domains are the Mori domains.

A domain is independent of finite character $\mathcal{F}$ (or an $\mathcal{F}-I F C$ domain) if it has a defining family $\mathcal{F}$ of primes that is independent and of finite character [9]. An ideal $I$ of a domain is called unidirectional if it belongs to a unique member of the defining family $\mathcal{F}$ of primes [9]. A domain $D$ is called a weakly Matlis domain if the intersection $D=\cap\left\{D_{P} \mid P \in \operatorname{Max}_{t}(D)\right\}$ is independent of finite character [10]. For an integral domain $D, X^{1}(D)$ will denote the set of height-one prime ideals of $D$. A domain $D$ is said to be a weakly Krull domain if $D$ is a locally finite intersection of its localizations at members of $X^{1}(D)$. A domain $D$ is called a Prüfer $v$-multiplication domain (PVMD) if every nonzero finitely generated ideal of $D$ is $t$-invertible [20].

Let $D$ be an integral domain and $I, J$ nonzero ideals of $D$. We say that $I, J$ is an almost condensed (AC) pair, if for each $0 \neq z \in I J$, there exists a pair $I^{\prime}, J^{\prime}$ of $\left(w\right.$-invertible) ideals of $D$ such that $I^{\prime} \subseteq I_{w}, J^{\prime} \subseteq J_{w}$ and $z D=\left(I^{\prime} J^{\prime}\right)_{w}$. We say that $D$ is an almost condensed $(A C)$ domain, if $I, J$ is an AC pair for all nonzero ideals $I$ and $J$ of $D$. If $I$ or $J$ is $w$-invertible (equivalently $t$-invertible), then $I, J$ is an AC pair (Remark 3). A domain $D$ is $\mathrm{AC}$ if and only if every pair of finitely generated ideals of $D$ is AC. Hence a PVMD is AC (Proposition 4). Since a Prüfer domain is a PVMD, studying $\mathrm{AC}$ domains covers a vast area of commutative ring theory of interest. An HC domain is clearly an AC domain. There exist simple examples of AC domains which are not $\mathrm{HC}$. For instance, the domain $\mathbb{Z}[X]$ is an $\mathrm{AC}$ domain but not HC. In general a non-Prüfer PVMD is an AC domain which is not HC. We show that every $t$-linked overring of an AC domain is $\mathrm{AC}$ (Proposition 2). If $D$ is an AC domain in which every maximal ideal is a $t$-ideal, then $D$ is an HC 
domain (Proposition 6). If $D$ is an $\mathrm{AC}$ domain with $C l_{t}(D)=0$, then every pair of $w$-ideals of $D$ is condensed (Proposition 8). If $D$ is an AC domain, then $D_{M}$ is condensed for each $M \in \operatorname{Max}_{t}(D)$ (Proposition 9). A domain $D$ is a PVMD if and only if it is integrally closed and AC (Proposition 10). If $D$ is an $\mathcal{F}$-IFC domain such that $D_{M}$ is condensed for each $M \in \mathcal{F}$, then $D$ is an AC domain (Proposition 12). A weakly Matlis domain $D$ is an AC domain if and only if $D_{M}$ is condensed for each $M \in \operatorname{Max}_{t}(D)$ (Corollary 13). A Noetherian domain $D$ is an AC domain if and only if $D_{M}$ is condensed for each $M \in \operatorname{Max}_{t}(D)$ if and only if $D$ is weakly Krull and $D_{M}$ is condensed for each $M \in X^{1}(D)$ (Theorem 14). If $\left\{D_{i}\right\}_{i \in \Lambda}$ is an ascending chain of AC domains such that $D_{i}$ is $t$-linked over $D_{j}$ for every $D_{j} \subseteq D_{i}$, then $D=\cup_{i \in \Lambda} D_{i}$ is an AC domain (Proposition 15). If $D$ is a Noetherian weakly Krull domain whose integral closure $D^{\prime}$ is two-generated as a $D$-module, then $D\left[\left\{X_{\lambda}\right\}\right]$ is an AC domain for every set of indeterminates $\left\{X_{\lambda}\right\}_{\lambda \in \Lambda}$ over $D$ (Proposition 16).

Throughout this paper all rings are (commutative unitary) integral domains.

For a domain $D$, denote the complete integral closure by $\bar{D}$ and the integral closure by $D^{\prime}$. Our standard reference for any undefined notation or terminology is [18].

Recall that an extension $R \subseteq T$ of domains is said to be $t$-linked if, whenever $J$ is a nonzero finitely generated ideal of $R$ with $J^{-1}=R$, we have $(J T)^{-1}=T$. Such extensions were introduced in [17]. In [6, Proposition 1.1], it is proved that every overring of an $\mathrm{HC}$ domain is $\mathrm{HC}$. In the next proposition, we shall prove that a $t$-linked overring of an AC domain is AC. A lemma is in order.

Lemma 1. Let $R \subseteq T$ be a t-linked extension of domains. Then $\left(I_{w} T\right)_{w}=$ $(I T)_{w}$ for any nonzero ideal $I$ of $R$.

Proof. Let $y \in I_{w}$. Then $y A \subseteq I$ for some finitely generated ideal $A$ with $A^{-1}=R$. This implies that $y A T \subseteq I T$ with $(A T)^{-1}=T$, because $T$ is a $t$-linked overring of $R$. Hence $y \in(I T)_{w}$ and thus $I_{w} \subseteq(I T)_{w}$. This implies that $\left(I_{w} T\right)_{w} \subseteq(I T)_{w}$. The opposite inclusion is clear.

Proposition 2. Let $D$ be an $A C$ domain and $E$ a t-linked overring of $D$. Then $E$ is also an $A C$ domain. In particular, every fraction ring of an $A C$ domain is $A C$.

Proof. Let $I, J$ be two nonzero ideals of $E$ and $0 \neq z \in I J$. Then $z=$ $x_{1} y_{1}+\cdots+x_{n} y_{n}$ for some $x_{i} \in I$ and $y_{i} \in J$. Let $0 \neq d \in D$ such that $d x_{i}, d y_{i} \in D$ for all $i$. Then $d^{2} z \in\left(d x_{1} D+\cdots+d x_{n} D\right)\left(d y_{1} D+\cdots+d y_{n} D\right)$. Since $D$ is $\mathrm{AC}, d^{2} z D=\left(I^{\prime} J^{\prime}\right)_{w}$ for some ideals $I^{\prime} \subseteq\left(d x_{1} D+\cdots+d x_{n} D\right)_{w}$ and $J^{\prime} \subseteq\left(d y_{1} D+\cdots+d y_{n} D\right)_{w}$. Then $I^{\prime \prime}=I^{\prime} / d \subseteq\left(x_{1} D+\cdots+x_{n} D\right)_{w}$, 
$J^{\prime \prime}=J^{\prime} / d \subseteq\left(y_{1} D+\cdots+y_{n} D\right)_{w}$ are fractional ideals of $D$ and $z D=\left(I^{\prime \prime} J^{\prime \prime}\right)_{w}$. This implies that $z E=(z E)_{w}=\left(\left(I^{\prime \prime} J^{\prime \prime}\right)_{w} E\right)_{w}$. Since $E$ is $t$-linked, we get that $z E=\left(I^{\prime \prime} J^{\prime \prime} E\right)_{w}=\left(\left(I^{\prime \prime} E\right)\left(J^{\prime \prime} E\right)\right)_{w}$ and $I^{\prime \prime} E \subseteq I_{w} E \subseteq\left(I_{w} E\right)_{w}=(I E)_{w}$, $J^{\prime \prime} E \subseteq J_{w} E \subseteq\left(J_{w} E\right)_{w}=(J E)_{w}$, cf. Lemma 1. The in particular statement follows from [7, Proposition 2.3(3)].

Remark 3. Let $D$ be a domain and $I, J$ be nonzero ideals of $D$. If $I$ or $J$ is $w$-invertible (equivalently t-invertible, cf. [3, Corollary 2.17]), then $I, J$ is an $A C$ pair. Indeed, if $J$ is $w$-invertible and $0 \neq z \in I J$, then $z D=\left(\left(z J^{-1}\right) J\right)_{w}$ with $z J^{-1} \subseteq I \subseteq I_{w}$.

Proposition 4. A domain $D$ is an $A C$ domain if and only if every pair of finitely generated ideals of $D$ is an $A C$ pair. In particular, every $P V M D$ is an $A C$ domain.

Proof. One implication is clear. For the converse, let $I, J$ be an arbitrary pair of ideals of $D$ and $0 \neq z \in I J$. Then $z=i_{1} j_{1}+\cdots+i_{n} j_{n}$, where $i_{k} \in I$ and $j_{k} \in J$ for $k=1, \ldots, n$. Then $z \in I^{\prime} J^{\prime}$, where the ideals $I^{\prime}$ and $J^{\prime}$ are given by $I^{\prime}=\left(i_{1}, \ldots ., i_{n}\right) \subseteq I$ and $J^{\prime}=\left(j_{1}, \ldots ., j_{n}\right) \subseteq J$. By hypothesis, $I^{\prime}, J^{\prime}$ is an AC pair, so there exist ideals $I^{\prime \prime} \subseteq I_{w}^{\prime} \subseteq I_{w}$ and $J^{\prime \prime} \subseteq J_{w}^{\prime} \subseteq J_{w}$ such that $z D=\left(I^{\prime \prime} J^{\prime \prime}\right)_{w}$. The "in particular" assertion follows from Remark 3 because a nonzero fractional ideal is $w$-invertible if and only if it is $t$-invertible.

Example 5. Consider the domain $D=F\left[\left[X^{2}, X^{3}\right]\right]$ where $F$ is a field. Then by [13, Example 2.3], $D$ is condensed domain and hence AC. Clearly $D$ is not $P V M D$ because it is not integrally closed.

Proposition 6. Let $D$ be an $A C$ domain in which every maximal ideal is a t-ideal. Then $D$ is an $H C$ domain.

Proof. If every maximal ideal is a $t$-ideal, then $w=d$, cf. [24, Proposition $2.2]$.

Example 7. Since a quasi-local HC domain is condensed [6], Proposition 6 shows that, a quasi-local one-dimensional domain is $A C$ if and only if it is condensed. So, the rings $\mathbb{R}+X \mathbb{R}(Y, Z)[[X]]$ and $\mathbb{R}\left[\left[X^{3}, X^{5}, X^{6}, X^{8}, \ldots\right]\right]$ are not $A C$, cf. [13, Example 2.11].

Proposition 8. Let $D$ be an $A C$ domain with $C l_{t}(D)=0$. Then every pair of $w$-ideals of $D$ is condensed.

Proof. Let $I, J$ be a pair of $w$-ideals of $D$ with $0 \neq z \in I J$. Since $D$ is AC, there exist $I^{\prime}, J^{\prime}$ (t-invertible) ideals of D such that $I^{\prime} \subseteq I_{w}=I, J^{\prime} \subseteq J_{w}=J$ and $z D=\left(I^{\prime} J^{\prime}\right)_{w}$. As $I^{\prime}, J^{\prime}$ are $w$-invertible and $C l_{t}(D)=0$, we get $I^{\prime}=x D$ and $J^{\prime}=y D$ for some $x \in I$ and $y \in J$. Then $z D=((x D)(y D))_{w}=((x y) D)_{w}=$ $(x y) D$ implies $z=x y u$ for some unit $u$. Thus $D$ is condensed. 
Proposition 9. Let $D$ be an $A C$ domain. Then $D_{M}$ is condensed for each $M \in \operatorname{Max}_{t}(D)$.

Proof. Let $M \in \operatorname{Max}_{t}(D)$ and $0 \neq z \in\left(I D_{M}\right)\left(J D_{M}\right)$, where $I, J$ are ideals of $D$. Then $s z \in I J$ for some $s \in D-M$. Since $D$ is AC, there exist $I^{\prime}, J^{\prime}$ ideals of $D$ such that $I^{\prime} \subseteq I_{w}, J^{\prime} \subseteq J_{w}$ and $s z D=\left(I^{\prime} J^{\prime}\right)_{w}$. Then $z D_{M}=\left(\left(I^{\prime} J^{\prime}\right)_{w}\right) D_{M}=\left(I^{\prime} J^{\prime}\right) D_{M}$ and $I^{\prime} D_{M} \subseteq I D_{M}, J^{\prime} D_{M} \subseteq J D_{M}$. As $I^{\prime} D_{M}$ and $J^{\prime} D_{M}$ are principal, $D_{M}$ is condensed.

In [6, Proposition 1.2], it is shown that an integral domain is Prüfer if and only if it is integrally closed and HC. We extend this result to AC domains.

Proposition 10. A domain $D$ is a PVMD if and only if it is integrally closed and an $A C$ domain.

Proof. A PVMD is integrally closed (cf. [22, Theorem 3.5]) and by Proposition 4 it is an AC domain. Conversely, assume that $D$ is integrally closed and an AC domain. Then by Proposition 9, [11, Main Theorem] and [23, Theorem 63], $D_{M}$ is a valuation domain for each maximal $t$-ideal $M$ of $D$. Hence $D$ is a PVMD, cf. [20, Theorem 5].

Corollary 11. The complete integral closure of an AC domain is a PVMD.

Proof. Let $D$ be an AC domain. Then $\bar{D}$ is $t$-linked by [7, Proposition 2.3(5)] and integrally closed by [18, Theroem 13.1(2)]. Now apply Propositions 2 and 10 .

Recall from [9] that a family $\mathcal{F}=\left\{P_{i}\right\}_{i \in L}$ of nonzero prime ideals of $D$ is called a defining family of primes for $D$ if $D=\bigcap_{i \in L} D_{P_{i}}$. If, further, every nonzero nonunit of $D$ belongs to at most finitely many members of $\mathcal{F}, \mathcal{F}$ is of finite character, and if no two members of $\mathcal{F}$ contain a nonzero prime ideal, $\mathcal{F}$ is independent. An ideal $I$ of a domain is called unidirectional if it belongs to a unique member of the defining family $\mathcal{F}$ of primes. An integral domain $D$ is independent of finite character $\mathcal{F}$ (or an $\mathcal{F}$-IFC-domain) if it has a defining family $\mathcal{F}$ of primes that is independent and of finite character. The corresponding family $\left\{D_{P_{i}}\right\}_{i \in L}$ of overrings of $D$ induces a star operation $*_{\mathcal{F}}$ on $D$ defined by $I \longmapsto I^{* \mathcal{F}}=\bigcap_{i \in L} I D_{P_{i}}$ for all $I \in F(D)$. We shall often refer to $*_{\mathcal{F}}$ as the star operation induced by $\mathcal{F}$. By [3, Corollary 2.13] and $[9$, Proposition 3.2], $I^{*^{\mathcal{F}}} \subseteq I_{w}$.

Proposition 12. Let $D$ be an $\mathcal{F}-I F C$ domain such that $D_{M}$ is condensed for each $M \in \mathcal{F}$. Then $D$ is an $A C$ domain. 
Proof. Let $I, J$ be nonzero ideals of $D$. Let $0 \neq x \in I J$ and let $M_{1}, \ldots, M_{n}$ be the members of $\mathcal{F}$ containing $x$. Since $I D_{M_{i}}, J D_{M_{i}}$ is a condensed pair, $x=$ $a_{i} b_{i}$ for some $a_{i} \in I D_{M_{i}}$ and $b_{i} \in J D_{M_{i}}$. Hence $x D_{M_{i}}=\left(A_{i}\right)_{M_{i}}\left(B_{i}\right)_{M_{i}}$, where $A_{i}=a_{i} D_{M_{i}} \cap D$ and $B_{i}=b_{i} D_{M_{i}} \cap D$ are unidirectional ideals with respect to $\mathcal{F}$ or equal to $D$, cf. [9, Theorem 3.3]. Set $A=A_{1} \cdots A_{n}$ and $B=B_{1} \cdots B_{n}$. We have $(A B)_{M_{i}}=\left(A_{i} B_{i}\right)_{M_{i}}=(x D)_{M_{i}}$. Also $(A B) D_{M}=D_{M}=x D_{M}$ for each $M$ in $\mathcal{F}$ distinct from $M_{1}, \ldots, M_{n}$. It follows that $(A B)^{* \mathcal{F}}=(x D)^{* \mathcal{F}}=x D$ and hence $x D=(A B)_{w}$ with $A \subseteq I^{* \mathcal{F}} \subseteq I_{w}$ and $B \subseteq J^{* \mathcal{F}} \subseteq J_{w}$.

Recall from [10], that a domain $D$ is called a weakly Matlis domain if the intersection $D=\cap\left\{D_{P} \mid P \in \operatorname{Max}_{t}(D)\right\}$ is independent of finite character.

Corollary 13. Let $D$ be a weakly Matlis domain. Then $D$ is an $A C$ domain if and only if $D_{M}$ is condensed for each $M \in \operatorname{Max}_{t}(D)$.

Proof. Apply Proposition 12 and 9.

Theorem 14. Let $D$ be a Noetherian domain. Then the following are equivalent:

(1) $D$ is an $A C$ domain.

(2) $D_{M}$ is condensed for each $M \in \operatorname{Max}_{t}(D)$.

(3) $D$ is weakly Krull and $D_{M}$ is condensed for each $M \in X^{1}(D)$.

Proof. $(1) \Rightarrow(2)$. Apply Proposition 9 .

$(2) \Rightarrow(3)$. As $D$ is a Mori domain, the intersection $D=\cap_{M \in \operatorname{Max}_{t}(D)} D_{M}$ has finite character, cf. [14, Theorem 3.3]. Also every maximal $t$-ideal $M$ of $D$ has height one. Indeed, if $h t(M)>1$, then $h t\left(M D_{M}\right)>1$ which is a contradiction because a Noetherian condensed domain has dimension at most one, cf. [13, Corollary 2.9]. Thus $\operatorname{Max}_{t}(D)=X^{1}(D)$ and we are done.

$(3) \Rightarrow(1)$. By $[8$, Lemma 2.1], every maximal $t$-ideal has height one. So $D$ is an $\mathcal{F}$-IFC domain with $\mathcal{F}=X^{1}(D)$. Hence by Proposition $12, D$ is an AC domain.

Proposition 15. Let $\left\{D_{i}\right\}_{i \in \Lambda}$ be an ascending chain of $A C$ domains such that each $D_{i}$ is t-linked over $D_{j}$ for $D_{j} \subseteq D_{i}$. Then $D=\cup_{i \in \Lambda} D_{i}$ is an $A C$ domain.

Proof. Let $I, J$ be two nonzero ideals of $D$ with $0 \neq z \in I J$. Then $z=$ $x_{1} y_{1}+\cdots+x_{n} y_{n}$, where $x_{k} \in I$ and $y_{k} \in J$ for $k=1, \ldots, n$. There exist $i_{0} \in \Lambda$ such that $D_{i_{0}}$ contains all elements $x_{i}, y_{j}$ and so $z \in D_{i_{0}}$. Let $I^{\prime}=$ $x_{1} D_{i_{o}}+\cdots+x_{n} D_{i_{o}}$ and $J^{\prime}=y_{1} D_{i_{o}}+\cdots+y_{n} D_{i_{o}}$. Then $z \in I^{\prime} J^{\prime}$. As $D_{i_{0}}$ is an AC domain, $z D_{i_{0}}=\left(I^{\prime \prime} J^{\prime \prime}\right)_{w}$ for some ideals $I^{\prime \prime} \subseteq I_{w}^{\prime}$ and $J^{\prime \prime} \subseteq J_{w}^{\prime}$. Since $D$ is t-linked over $D_{i_{0}}$ (cf. [7, Proposition 2.3(1)]), $z D=\left(\left(I^{\prime \prime} J^{\prime \prime}\right)_{w} D\right)_{w}=$ $\left(I^{\prime \prime} J^{\prime \prime} D\right)_{w}=\left(\left(I^{\prime \prime} D\right)\left(J^{\prime \prime} D\right)\right)_{w}$ and $I^{\prime \prime} D \subseteq\left(I^{\prime} D\right)_{w} \subseteq I_{w}, J^{\prime \prime} D \subseteq\left(J^{\prime} D\right)_{w} \subseteq J_{w}$, cf. Lemma 1. 
A domain $D$ is said to have the two-generator property or simply is twogenerated if every ideal of $\mathrm{D}$ is generated by two elements.

Proposition 16. Let $D$ be a Noetherian weakly Krull domain whose integral closure $D^{\prime}$ is two-generated as a D-module. Then $D\left[\left\{X_{\lambda}\right\}\right]$ is an AC domain for every set of indeterminates $\left\{X_{\lambda}\right\}_{\lambda \in \Lambda}$ over $D$.

Proof. Since a polynomial extension is $t$-linked [22, Corollary 2.3], we can assume that $\left\{X_{\lambda}\right\}_{\lambda \in \Lambda}$ is finite, cf. Proposition 15. By induction, on the cardinality of $\Lambda$, we can assume that $\left\{X_{\lambda}\right\}_{\lambda \in \Lambda}$ has a single indeterminate $X$. The domain $D[X]$ is a Noetherian weakly Krull domain (cf. [7, Corollary 4.12]) and $D^{\prime}[X]$ is two-generated as a $D[X]$-module. Let $P$ be a height-one prime ideal of $D[X]$. By using $(a) \Leftrightarrow(c)$ in $\left[19\right.$, Theorem 2.3], $D[X]_{P}$ is twogenerated and by $[6$, Corollary 1.5] it is condensed. By Theorem $14, D[X]$ is an AC domain.

Example 17. Let $A \subset B$ be an extension of $D V R$ s such that $B_{A-\{0\}}$ is a field, $q f(A) \cap B=A$ and $B$ is two-generated as an $A$-module. Then $D=A+X B[X]$ is an $A C$ domain. Indeed, $D$ is a Noetherian weakly Krull domain (see [12, Theorem 3.4]) and $D^{\prime}=B[X]$ is two-generated as a D-module. In particular, the domain $\mathbb{R}[[X]]+Y \mathbb{C}[[X]][Y]$ is an $A C$ domain.

Remark 18. A domain $D$ is PVMD if and only if $D[X]$ is PVMD, cf. [22, Theorem 3.7]. Therefore an integrally closed domain $D$ is $A C$ if and only $D[X]$ is $A C$, cf. Proposition 10.

Recall from [18, Section 33] that the Nagata ring $D(X)$ of a domain $D$ is the fraction ring of $D[X]$ with respect to the multiplicative set $S$ of polynomials whose coefficients generate the unit ideal. Our final example shows that if $D$ is a Noetherian AC domain, then $D[X]$ need not be an $\mathrm{AC}$ domain.

Example 19. Let $\mathbf{B}$ be the field of all complex numbers which can be constructed by straight-edge and compass from 0 and 1 (see [21, page 210]). Let $D=\mathbf{B}+X \mathbf{B}(\sqrt[3]{3})[[X]]$. Then $D$ is a local condensed domain (see $[4$, Example 2.11]) and $D^{\prime}=\mathbf{B}(\sqrt[3]{3})[[X]]$ is a DVR which is finite over $D$. The domain $D[Y]$ is a Noetherian weakly Krull domain, cf. [12, Theorem 3.4]. Let $M=X \mathbf{B}(\sqrt[3]{3})[[X]]$. Then $D[Y]_{M[Y]}=D(Y)$. The ring $D(Y)$ is a local ring with residue field $\mathbf{B}(Y)$. Also the integral closure of $D(Y)$ is $D^{\prime}(Y)$ (see [18, Exercise 2, p. 415]) which is a DVR and is finite over $D(Y)$. The residue field of $D^{\prime}(Y)$ is $\mathbf{B}(\sqrt[3]{3})(Y)$ and $[\mathbf{B}(\sqrt[3]{3})(Y): \mathbf{B}(Y)]=3$. Note that $Z^{2}-Y$ is a quadratic polynomial in $\mathbf{B}(Y)[Z]$ which does not split in $\mathbf{B}(Y)$. By [5, Theorem 5], $D(Y)$ is not condensed and hence $D[Y]$ is not AC, cf. Theorem 14. 
Acknowledgements. We would like to express our sincere thanks to Abdus Salam School of Mathematical Sciences, GC University Lahore and HEC(Higher Education Commission of Pakistan) for their support in this research work. We thank Professor Muhammad Zafrullah who read an earlier version of this paper and sent us useful comments. Our sincere thanks to Tiberiu Dumitrescu for many useful email discussions. We also appreciate the valuable suggestions and changes recommended by the referees.

\section{References}

[1] D.D. Anderson, Star-operations induced by overrings, Comm. Algebra 16 (1988), 2535-2553.

[2] D.D. Anderson and D.F. Anderson, Some remarks on star operations and the class group, J. Pure Appl. Algebra 51 (1988), 27-33.

[3] D.D. Anderson and S.J. Cook, Two star operations and their induced latices, 28 (2000), 2461-2475

[4] D.D. Anderson and T. Dumitrescu, Condensed domains, Canad. Math. Bull. 46 (2003), 3-13.

[5] D.D. Anderson and T. Dumitrescu, Condensed local domains which are not strongly condensed, Math. Rep. 55 (2003), 205-209.

[6] D.D. Anderson and T. Dumitrescu, Half condensed domains, Houston J. Math. 30 (2004), 929-936.

[7] D.D. Anderson, E.G. Houston, M. Zafrullah, $t$-linked extensions, the $t$ class group, and Nagata's theorem, J. Pure Appl. Algebra 86 (1993), 109-124.

[8] D.D. Anderson, J. L. Mott and M. Zafrullah, Finite character representation of integral domains, Bollettino U.M.I. 76-B (1992), 613-630.

[9] D.D. Anderson and M. Zafrullah, Independent locally-finite intersections of localizations, Houston J. Math. 25 (1999), 433-452.

[10] D.D. Anderson and M. Zafrullah, Splitting sets and weakly Matlis domains, Proceedings of the Fifth International Fez Conference on Commutative Algebra and its Applictions, Fez, Morocco, 2008.

[11] D.F. Anderson, J.T. Arnold and D.E. Dobbs, Integrally closed condensed domains are Bézout, Canad. Math. Bull. 28 (1985), 98-102. 
[12] D.F. Anderson, G.W. Chang, A.J. Park, Weakly Krull and related domains of the form $D+M, A+X B[X], A+X^{2} B[X]$, Rocky Mountain J. Math. 36(2006), 1-22.

[13] D.F. Anderson and D.E. Dobbs, On the product of ideals, Canad. Math. Bull. 26 (1983), 106-114.

[14] V. Barucci, Mori domains, Non-Noetherian Commutative Ring Theory (S.C Chapman and S. Glaz Editors), Math. Appl. Kluwer Acad. Publ. Dordrecht, 520(2000), 57-73.

[15] A. Bouvier and M. Zafrullah, On some class group of an integral domain, Bull. Soc. Math. Greece 29 (1988), 45-59.

[16] G.W. Chang, Strong Mori domains and the ring $D[x]_{N_{v}}$, J. Pure Appl. Algebra 197 (2005), 3669-3686.

[17] D. Dobbs, E. Houston, T. Lucas, M. Zafrullah, t-Linked overrings and Prüfer v-multiplication domains, Comm. Algebra 17 (1989) 28352852.

[18] R. Gilmer, Multiplicative Ideal Theory, Marcel Dekker, New York, 1972.

[19] C. Greither, On the two generator problem for the ideal of a onedimensional ring, J. Pure Appl. Algebra 24 (1982), 265-276.

[20] M. Griffin, Some results on $v$-multiplications rings, Canad. J. Math. 19 (1967), 710-722.

[21] N. Jacobson, Basic Algebra I, Freeman, San Francisco, 1974.

[22] B.G. Kang, Prüfer v-multiplication domains and the ring $R[X]_{N_{v}}$, J. Algebra 123 (1989), 151-170.

[23] I. Kaplansky, Commutative Rings, Revised Edition, The University of Chicago Press, Chicago and London, 1974.

[24] A. Mimouni, Integral domains in which each ideal is a $w$-ideal, Comm. Alg. 33(2005), 1345-1355.

Waseem Khalid,

Department of Mathematics,

Govt. College University,

Faisalabad, Pakistan.

Email: wsmkhld@yahoo.com

Shafiq Ur Rehman,

Department of Mathematics,

COMSATS Institute of Information Technology,

Attock, Pakistan.

Email: shafiq_ur_rahman2@yahoo.com 